\title{
Uma abordagem prática para o ensino do eletromagnetismo usando um motor de indução de baixo custo
}

\author{
A practical approach to the teaching of electromagnetism using low cost induction motor \\ Alexandre Magno Ferreira Diniz ${ }^{*} \sqrt{0}$, Rômulo Diniz Araújo ${ }^{1}$ \\ ${ }^{1}$ Instituto Federal de Educação, Ciência e Tecnologia do Ceará, Juazeiro do Norte, CE, Brasil
}

Recebido em 18 de July, 2018. Revisado em 30 de July, 2018. Aceito em 07 de August, 2018.

\begin{abstract}
O artigo descreve o desenvolvimento e construção de um motor aberto de indução trifásico de baixo custo para o ensino do eletromagnetismo nas disciplinas de física e máquinas elétricas de cursos técnicos. Na construção do protótipo foram usados materiais de sucata de estabilizador de tensão. Os computadores atuais possuem fontes internas chaveadas com tecnologia avançada que dispensa o uso dos módulos estabilizadores de tensão. Com isso, muitos desses módulos não são mais usados, tornando-se sucatas e gerando problemas de descarte para o meio ambiente. $\mathrm{O}$ motor foi implementado e funciona satisfatoriamente para a finalidade a que se destina. $\mathrm{O}$ protótipo desenvolvido é usado nas aulas práticas de eletromagnetismo e de máquinas elétricas dos cursos técnico e tecnológico em automação industrial. Nessas aulas são abordados diversos fenômenos do eletromagnetismo que o motor de indução experimenta.
\end{abstract}

Palavras-chave: Campo magnético, corrente induzida, motor de indução, experimentos em eletromagnetismo.

The paper describes the development and construction of a low-cost three-phase induction open motor for the teaching of electromagnetism in the disciplines of physics and electrical machines of the technical courses. Voltage stabilizer scrap materials were used in the construction of the prototype. Today's computers have built-in power supplies with advanced technology that eliminates the use of voltage stabilizer modules. As results, many of these modules are no longer used, becoming scrap and generating disposal problems for the environment. The motor was implemented and works satisfactorily for the intended purpose. The prototype is used in the practical classes of electromagnetism and electric machines of the technical level courses in electrotechnology and technology in industrial automation. In these classes several phenomena of electromagnetism related with the induction motor are discussed.

Keywords: Magnetic field, induced current, induction motor, electromagnetism experiments..

\section{Introdução}

O uso dos conjuntos didáticos como metodologia de ensino tem demonstrado melhoria no processo ensinoaprendizagem. Pinto et al. [1] comentam que as aulas práticas de laboratórios didáticos despertam nos alunos a vontade de aprender e os resultados se traduzem na melhoria significativa na qualidade do ensino. Corroborando, [2] e [3] explicam que as atividades desenvolvidas com a prática em ciência nos laboratórios minimizam as dificuldades do aluno, incentiva a investigação científica, instiga ao aluno a comprovação e verificação dos fenômenos físicos que se apresenta no dia-a-dia, auxiliando no entendimento dos conceitos abordados. Como proposta pedagógica para engajar e atrair a atenção dos alunos nas disciplinas e reduzir a evasão escolar nas escolas técnicas, [4] acreditam que a melhoria na qualidade das

*Endereço de correspondência: alexdinizeng@gmail.com aulas práticas de laboratório pode ser uma das soluções para o problema.

Certamente, os conjuntos didáticos que equipam os laboratórios são indispensáveis para o bom desenvolvimento dos cursos técnicos. No mercado, são encontrados diversos conjuntos didáticos de motor de indução aberto para o ensino do eletromagnetismo e de máquinas elétricas. Porém, os preços elevados desses conjuntos são restrições para aquisição ou mesmo atualização dos laboratórios de muitas escolas técnicas públicas. A proposta do uso do motor aberto de indução nas aulas práticas de física se apresenta como uma alternativa atraente, de baixo custo e viável para incrementar o estudo prático do estudo do eletromagnetismo, além de demonstrar com melhor propriedade os efeitos do eletromagnetismo que o motor experimenta.

A proposta do protótipo é a construção do motor usando sucata de material conseguida de estabilizadores de tensão. Os primeiros estabilizadores de tensão foram 
produzidos para a regulação da tensão nos televisores, aparelhos de rádio e geladeiras em 1940. Nesta época, esses aparelhos utilizavam válvulas para controlar a tensão com ganho de amplificação. Com a evolução da eletrônica, as válvulas foram substituídas pelos dispositivos eletrônicos de estado sólido como diodos e transistores, produzidos de material semicondutor.

Até há pouco tempo, os computadores eram ligados a esses estabilizadores de tensão para garantir o seu bom funcionamento. O estabilizador de tensão mais comum, encontrado nos escritórios ou nas casas, é construído com peças eletrônicas e por um transformador monofásico que reduz a tensão da rede elétrica para uma tensão adequada, de operação para o computador. Todavia, os atuais computadores possuem fonte interna de tensão chaveada com tecnologia avançada que dispensa o uso dos módulos estabilizadores de tensão. Com isso, muitos dos módulos antigos não são mais usados, tornando-se sucatas e gerando problemas de descarte para o meio ambiente. O lixo tecnológico, incluindo também como fonte geradora do lixo os equipamentos de informática, é responsável pela geração de centenas de toneladas de resíduos que, muitas vezes, é descartado em aterros sanitários e sem nenhum tratamento, agravando a degradação do meio ambiente [5].

Em termos gerais, o transformador é uma máquina elétrica construída por um núcleo de chapas finas de material ferromagnético e de duas ou mais bobinas de fios de cobre esmaltado enroladas neste núcleo, colocadas diametralmente opostas. Não existe ligação entre as duas bobinas, ou seja, elas estão eletricamente isoladas. Da mesma forma de construção do transformador, o motor de indução possui um estator, de núcleo formado com os mesmos materiais do transformador. A principal diferença entre as duas máquinas elétricas é o rotor do motor, que não existe no transformador. No motor, as bobinas são enroladas tanto no estator como no rotor. Em alguns tipos de motores, o rotor é construído com

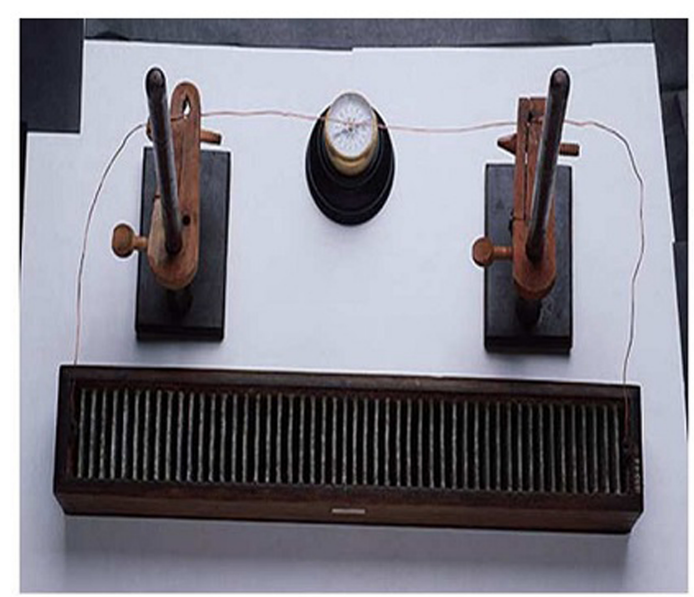

barras condutoras que fazem as mesmas funções dos fios nas bobinas. A interação eletromagnética entre o estator e rotor é feita por tensão induzida, sem nenhum contato físico entre as duas partes, girante e fixa.

O artigo descreve o desenvolvimento de um protótipo para o motor aberto de indução trifásico construído com material de sucata de estabilizador de tensão. O protótipo visa contribuir no processo de melhoria da aprendizagem aplicada aos alunos dos cursos de nível médio e técnico das disciplinas de física (eletricidade e eletromagnetismo) e de máquinas elétricas, com a proposta em abordar os conceitos e formalismos básicos dos fenômenos eletromagnéticos que o motor de indução experimenta.

\section{O eletromagnetismo aplicado ao motor de indução}

Os trabalhos sobre o campo magnético e eletromagnético iniciaram com os experimentos de Oersted, com os resultados publicados em 1820 . Oersted constatou a relação que existe entre a eletricidade e o magnetismo [6], e baseados nesses experimentos surgiram importantes trabalhos desenvolvidos por Ampère, Faraday, Maxwell, Tesla, e muitos outros pesquisadores. A Fig. 1a mostra o experimento de Oersted, sendo composto de uma bateria ligada a um fio de cobre suspenso, e uma bússola colocada em baixo do fio que é usada para indicar a existência e o sentido do campo magnético gerado pela passagem da corrente elétrica $i$.

A Fig. 1b faz um esboço das linhas de forças e do campo magnético criado pelo fluxo da corrente no fio. Além disso, pode-se observar que na área do campo magnético, as linhas de forças não se cruzam, e quando a corrente desce no fio o sentido do campo magnético é horário.

Logo em seguida a descoberta de Oersted, Biot e Savart formularam em 1820 uma equação para encontrar a magnitude do campo magnético a uma distância $r$ de

Figura 1: Estudo do campo magnético. (a) Materiais dos experimentos de Oersted, (b) desenho esquemático original. Fonte: WwW.en.wikipedia.org/wiki/Nikola_Tesla. 
um condutor retilíneo [7], expressa por

$$
\vec{B}=\frac{\mu \cdot i}{2 . \pi \cdot r}
$$

em que $\mu$ é a permeabilidade magnética do meio no interior da bobina em $\mathrm{Wb} / \mathrm{A} . \mathrm{m}, i$ é a corrente no fio em A, e $r$ é o raio em $\mathrm{m}$.

Outro experimento importante para o desenvolvimento do motor de indução foi à concepção de bobinas. Quando um fio é enrolado sobre um núcleo de material de alta permeabilidade magnética (material ferromagnético), formando uma bobina, o fluxo de corrente gera no centro da bobina um campo magnético linear, concentrado e forte.

A Fig. 2 mostra um esboço de uma bobina sem o núcleo de ferromagnético. $\mathrm{O}$ fluxo magnético linear no centro da bobina dá origem às polaridades norte e sul nas extremidades da bobina [8].

Na Fig. 2, é possível observar que o fluxo de corrente em cada condutor forma um campo circular próprio. A regra da mão direita a partir da lei de Ampère determina o sentido e a intensidade do campo magnético. Por convenção, a indicação • nos fios da parte superior significa que o sentido da corrente está saindo do papel e o sentido do campo magnético é anti-horário, e com a indicação $\mathbf{x}$ a corrente está entrando no papel e o sentido do campo magnético é horário. Como se pode notar, o sentido do campo magnético dos condutores superiores é oposto ao sentido do campo dos condutores inferiores. A interação entre os dois campos em cada segmento do condutor

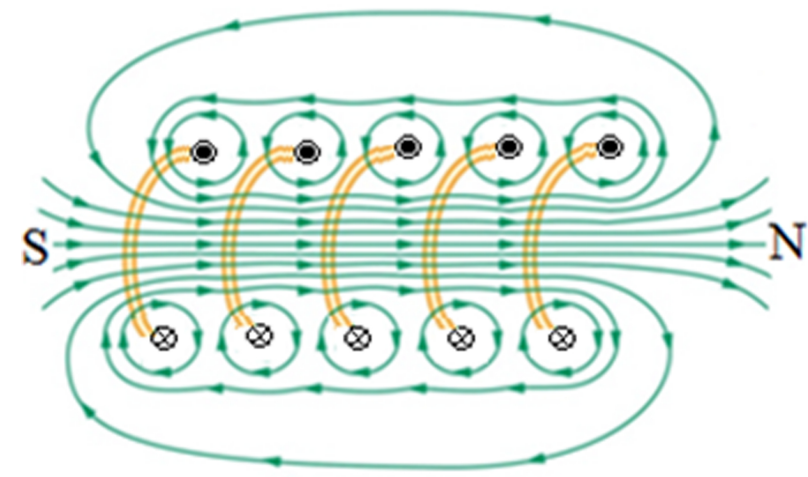

Figura 2: Direção e sentido do fluxo magnético gerado pela bobina. Fonte: [8]. gera um campo resultante linear no centro da bobina. A intensidade do campo magnético em Tesla no interior da bobina é determinada por

$$
\vec{B}=\mu \cdot i \frac{N}{L}
$$

onde $N / L$ é o número de espiras por unidade de comprimento $(\mathrm{m})$.

No estator do motor, a bobina energizada com corrente alternada gera um campo magnético resultante, fazendo com que os polos e o fluxo magnético mudem de intensidade e de sentido. Na prática, a bobina é bipartida, dividida igualmente em duas outras bobinas. A Fig. 3 mostra o sentido instantâneo do campo do magnético e as algumas linhas de força que formam o eixo magnético.

No momento da inversão da corrente, ocorre a mudança do sentido do campo magnético e da posição da polaridade da bobina. Portanto, o campo magnético no espaço do entreferro é variável no tempo, e pode ser considerada uniforme no espaço para o estudo básico do motor de indução. A equação definida para a densidade de campo magnético instantâneo é

$$
B(t)=B_{o} \cdot \cos (2 \cdot \pi \cdot f \cdot t+\theta)
$$

onde $B_{o}$ é a amplitude do campo magnético em T, $f$ é a frequência da rede elétrica em $\mathrm{Hz}, t$ é o tempo em s, e $\theta$ é o ângulo de defasagem em rad.

No motor de indução trifásico, o estator é formado por três bobinas bipartidas distribuídas uniformemente ao longo do perímetro do núcleo de ferromagnético do estator. Essas bobinas são energizadas com correntes trifásicas e o sistema de equações é determinado como

$$
\begin{array}{r}
B_{A}(t)=B_{o} \cdot \cos (2 . \pi \cdot f \cdot t) \\
B_{B}(t)=B_{o} \cdot \cos \left(2 . \pi \cdot f \cdot t+\frac{2 . \pi}{3}\right) \\
B_{C}(t)=B_{o} \cdot \cos \left(2 . \pi . f . t-\frac{2 . \pi}{3}\right)
\end{array}
$$

em que os subscritos $A, B$ e $C$ são usados para nomear as bobinas do motor trifásico. A Fig. 4 apresenta as curvas de campo magnético ao longo do tempo, geradas pelas bobinas.

Na Fig. 4, pode-se observar que as curvas são equilibradas e defasadas com o ângulo de $2 \pi / 3$ rad entre si, pois as correntes alternadas trifásicas que alimentam o

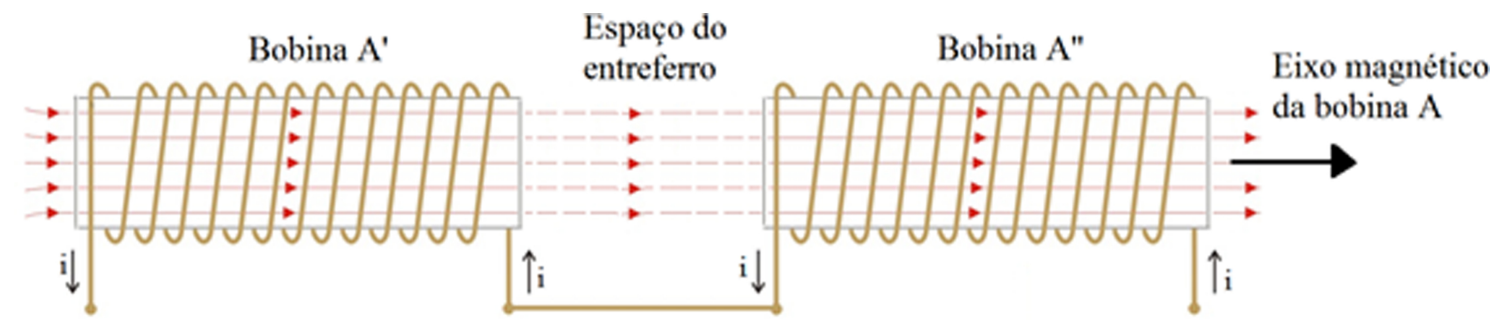

Figura 3: Disposição das bobinas enroladas no núcleo de ferromagnético. 
sistema são, também, equilibradas e defasadas com os mesmos ângulos.

As correntes induzidas nas bobinas foram descobertas por Faraday, em 1831, onze anos depois dos primeiros experimentos de Orested. Nos experimentos de Faraday foi observado que variando o fluxo magnético próximo a uma bobina é produzida uma tensão elétrica induzida que, por sua vez, dá origem a corrente induzida [9]. A lei de indução de Faraday para a tensão induzida é formulada como segue

$$
\epsilon=-N \frac{d \Phi}{d t}
$$

e, por sua vez

$$
\Phi=N \cdot B \cdot A \cdot \cos (2 \cdot \pi \cdot f \cdot t)
$$

em que $N$ é o número de voltas da bobina, $\Phi$ é o fluxo magnético em $\mathrm{Wb}$, e $A$ é a área da superfície da bobina que o fluxo atravessa em $\mathrm{m}^{2}$.

As correntes induzidas são observadas nos transformadores e nos motores de indução. No transformador, a bobina que está energizada pela fonte de tensão produz o fluxo magnético que por sua vez gera uma corrente induzida na bobina que alimenta a carga. No motor, as bobinas do estator produzem campo magnético com linhas forças que cortam as bobinas do rotor, e nessas bobinas geram as correntes induzidas. Também, as correntes induzidas geram o seu próprio campo magnético e a interação entre esses dois campos magnéticos produz um torque nos condutores, resultando no movimento do rotor no mesmo sentido de rotação do campo magnético.

O torque magnético pode ser conseguido colocando um dipolo magnético na forma de espira, dentro de uma região de um campo magnético com intensidade adequada as dimensões da espira. A Fig. 5 mostra a espira imersa no campo magnético, e os sentidos da corrente, dos vetores da força que atuam na espira, e que proporciona a rotação da espira.

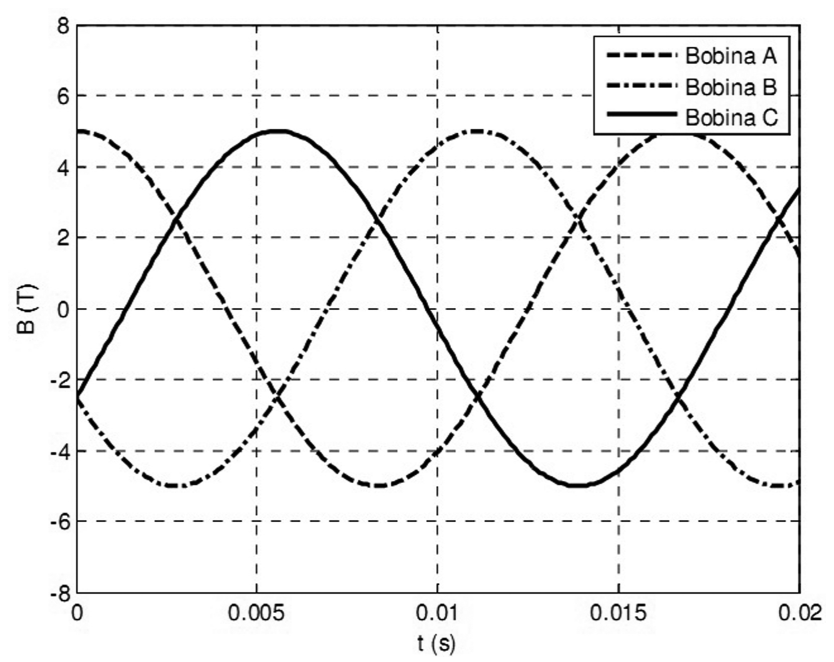

Figura 4: Curvas temporais de campo magnético nas bobinas.

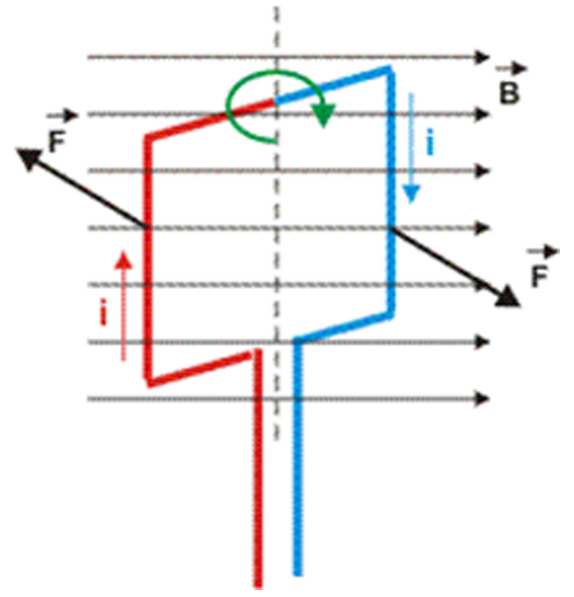

Figura 5: Obtenção do torque sobre uma espira. Fonte: [10].

O torque aplicado sobre o momento de dipolo magnético associado às espiras condutoras presas ao eixo do rotor quando imersas no campo magnético oriundo das bobinas do estator faz o eixo de um motor girar. O dipolo magnético fica sujeito a um torque que tende a fazê-lo girar. A expressão para o torque induzido na unidade N.m é

$$
\tau=\vec{F} \cdot r
$$

em que $\vec{F}$ é a força aplicada na espira em $\mathrm{N}$ e $r$ é o raio da espira em $\mathrm{m}$.

\section{Construção do motor de indução}

Basicamente, o motor de indução é composto por duas partes: o estator e o rotor. O estator é a parte fixa do motor, que recebe a alimentação da rede elétrica em seus terminais de ligação. O número de terminais de motores de indução trifásicos comerciais mais comuns varia de 6 a 12, conforme valores de tensões disponíveis na rede elétrica. Neste projeto específico, o motor tem seis terminais para a ligação de suas bobinas, com opções para ser ligado em delta (tensão mais baixa) ou em estrela (tensão mais alta).

As bobinas do estator são alimentadas por correntes e tensões trifásicas senoidais defasadas de $120^{\circ}$ elétricos entre si, que criam um campo magnético girante que varrem o rotor [10]. A Fig. 6 apresenta o projeto do motor de indução com seis bobinas e no centro encontrase o rotor. Esse projeto é baseado no primeiro motor desenvolvido em 1988 por Nikola Tesla.

A outra parte do motor é o rotor que está conectado ao eixo do motor, e recebe correntes induzidas vindas das bobinas do estator. No caso especial do protótipo desenvolvido, em que o rotor é construído com uma única massa metálica, as correntes induzidas na massa metálica são chamadas de correntes parasitas, também conhecidas por correntes de Foucault. É bom salientar que nos motores comerciais essas correntes são indesejáveis, pois provocam perdas no núcleo do rotor e reduz a eficiência 


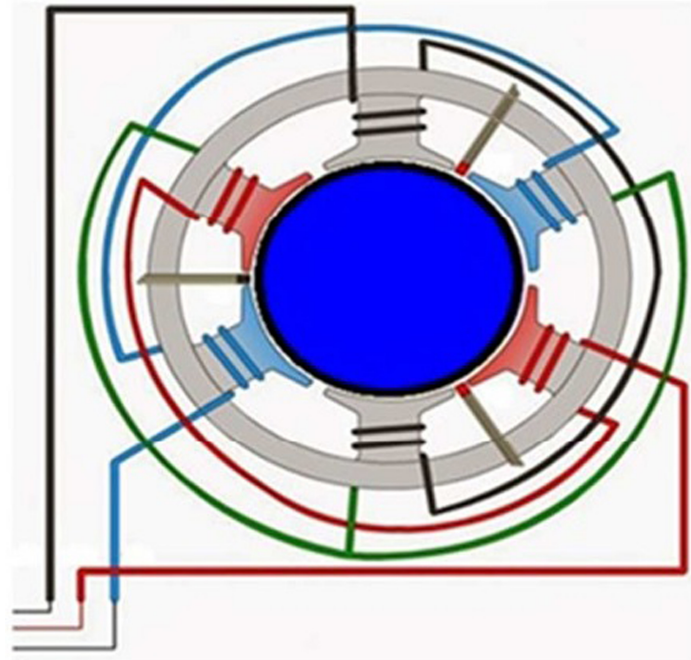

(a)

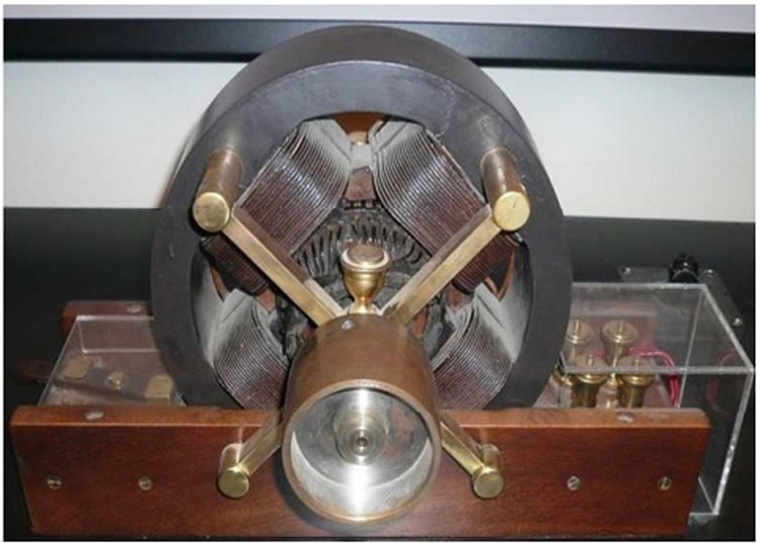

(b)

Figura 6: (a) Esquema do motor de indução com seis terminais; (b) primeiro motor de indução. Fonte: www.en.wikipedia.org/ wiki/Nikola_Tesla

do motor [11]. Nesses motores comerciais, o rotor tem bobinas com fios de cobre esmaltado ou barras condutoras de cobre ou alumínio, e a grande parte das correntes induzidas circula nessas bobinas.

\subsection{O estabilizador de tensão}

O estabilizador foi desenvolvido em 1940 com o objetivo de fazer a regulação da tensão de aparelhos movidos a válvulas, como os primeiros televisores e geladeiras que, diferentemente dos equipamentos atuais, possuem circuitos eletrônicos construídos com dispositivos de estado sólido (transistores, diodos e circuitos integrados) e eletromagnéticos. A Fig. 7 mostra um típico estabilizador de tensão e com a carcaça aberta, usados em escritórios e residências.

A função do estabilizador é garantir que o aparelho conectado a tomada de saída do estabilizador não perceba as possíveis alterações de tensão na rede elétrica. No momento da ocorrência de uma sobretensão, o estabi-

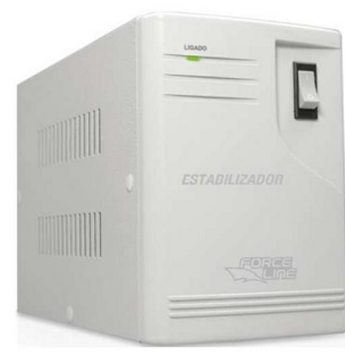

(a)

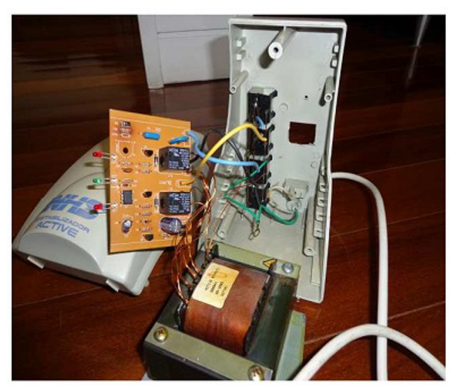

(b)
Figura 7: (a) Estabilizador de tensão comercial, (b) circuito eletrônico e transformador do estabilizador de tensão. lizador atua e busca regular a tensão de saída, evitando com isso o desgaste prematuro ou danos no aparelho. Por outro lado, quando ocorre uma subtensão na rede o estabilizador percebe e aumenta a tensão, garantindo a permanência do aparelho ligado [12]. Para tanto, o estabilizador possui fusíveis para a proteção contra curtocircuito e varistores que atuam como limitador de tensão. Os equipamentos e aparelhos eletrônicos mais modernos possuem fontes de alimentação que atuam automaticamente em redes de tensões $127 \mathrm{~V}$ ou $220 \mathrm{~V}$, realizando melhor a função dos estabilizadores. Os estabilizadores de tensão usados no projeto possuem uma placa eletrônica, um transformador abaixador, fusíveis de proteção, e terminais de tomadas para a conexão de terminais.

As principais especificações do estabilizador de tensão monofásico são a tensão de saída, variação de tensão de entrada e de saída, fator de potência, potência aparente do equipamento, rendimento, e tempo de resposta. Geralmente, a variação de tensão de entrada máxima é de $\pm 15 \%$ e a de saída é $\pm 3 \%$, e o tempo de resposta é de pelo menos $8,3 \mathrm{~ms}$. Também, existem estabilizadores trifásicos que podem ter proteção contra a falta de fase.

\subsection{Montagem do estator}

O estator é formado pelo núcleo de ferromagnético e por bobinas fixadas nas ranhuras do núcleo. Na construção do núcleo do estator foram utilizados os núcleos dos transformadores aproveitados das sucatas dos estabilizadores de tensão. O núcleo do transformador é do tipo envolvente, formado por duas peças, sendo uma chapa no formato "E" e outra no formato "I". A Fig. 8 apresentam o transformador completo com as bobinas e terminais de ligação, e as peças do transformador desmontado. 


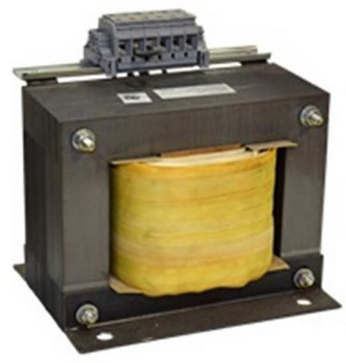

(a)

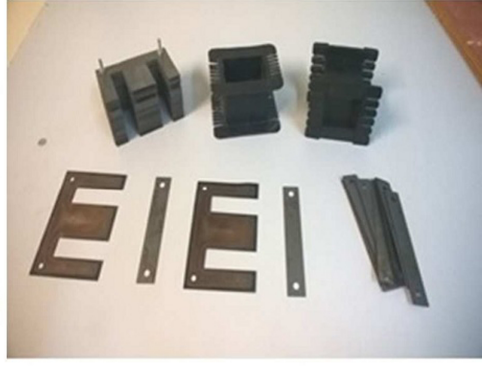

(b)
Figura 8: (a) Transformador monofásico; (b) chapas de ferromagnético e carretéis das bobinas.

O processo de construção do estator inicia-se com a retirada da bobina da parte central do núcleo, em seguida são desmontadas todas as chapas. Para construir o núcleo do protótipo do motor, foram unidas por meio de parafusos e porcas seis peças "E" com as peças "I", formando no final da montagem a figura geométrica do dodecágono (12 lados). O aspecto do estator pronto é mostrado na Fig. 9.

As bobinas foram calculadas considerando a densidade de campo magnético de $8.000 \mathrm{~T}$, valor padrão para material com $2 \%$ de silício, e um transformador de potência de 300 VA que foi retido do estabilizador. O número de voltas de fios das bobinas é calculado pela Equação [13]

$$
N=\frac{V \cdot 10^{8}}{4,44 \cdot f \cdot S_{L} \cdot B}
$$

onde $V$ é tensão na bobina em $\mathrm{V}, S_{L}$ é a seção líquida do núcleo em $\mathrm{cm}^{2}$, e $B$ é a densidade magnética em $\mathrm{G}$. A seção líquida é o produto do comprimento do lado A e B, conforme mostrado na Fig. 10.

A seção do fio das bobinas é calculada a partir da equação da corrente elétrica em função da potência aparente (VA), da tensão do lado primário e do fator de potência [14]. A corrente da bobina do primário do transformador

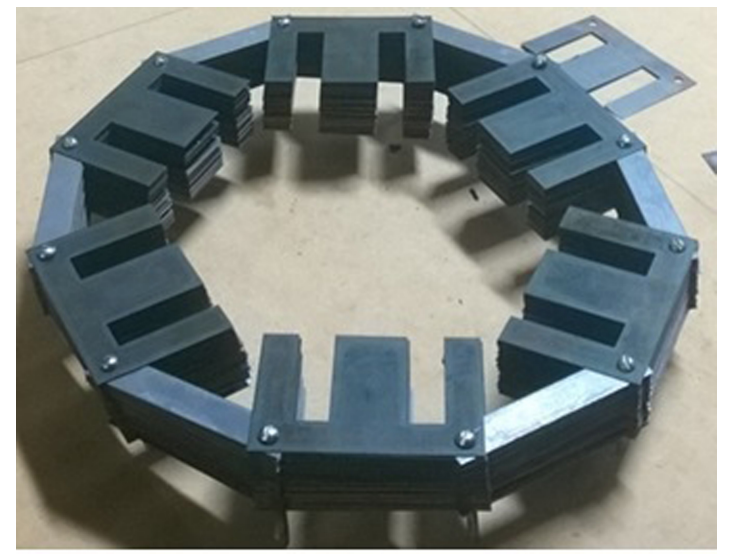

Figura 9: Núcleo do estator do motor de indução finalizado.

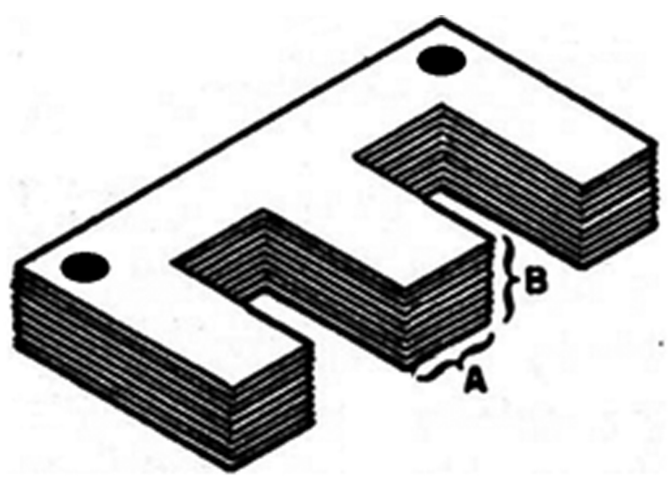

Figura 10: Ilustração da área da seção líquida do núcleo. Fonte: [13].

é dada por

$$
I_{p}=\frac{S}{U_{p} \cdot F P}
$$

onde $S$ é a potência aparente do transformador em VA, e FP é o fator de potência.

Uma bobinadeira foi criada usando um motor de indução conectado a um conversor de frequência como mostrado na Fig. 11.

O conversor de frequência que muitas vezes é chamado de inversor é usado para reduzir a velocidade de rotação do eixo de tal forma que foi possível realizar com segurança o serviço de bobinagem. Também, foi desenvolvido um contador de voltas com uma simples calculadora, uma chave de acionamento magnético (reed switch) e um ímã. O circuito da chave magnética foi conectado a tecla "+" da calculadora, e cada vez que o ímã que está fixado na bobina, passa enfrente a chave magnética, a calculadora soma uma unidade, possibilitando realizar a contagem com rapidez e sem erros.

\subsection{Construção do rotor}

A tipologia de construção do rotor para o motor de indução comercial consiste de dois tipos. O rotor em gaiola de esquilo (o mais comum) e o de rotor bobinado. Em geral,

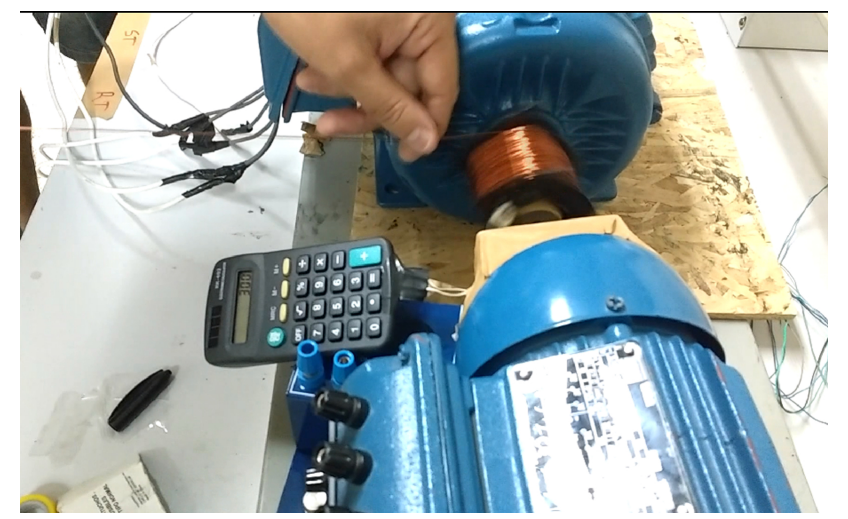

Figura 11: Bobinadeira e contador de voltas desenvolvidas para o protótipo. 
a escolha do tipo de rotor se baseia na característica da carga acoplada ao eixo e de como é realizada a partida do motor. A Fig. 12 ilustra o comparativo entre os dois rotores comerciais.

$\mathrm{Na}$ intenção de reduzir os custos do projeto foi usada uma lata cilíndrica metálica para a construção do rotor. Diversos cilindros de diâmetros e alturas diferentes foram testados para o rotor. A Fig. 13 apresenta um teste do rotor usando uma lata usada para condicionamento de doces.

O rotor confeccionado tem diâmetro de $15,5 \mathrm{~cm}$, adequado às dimensões do diâmetro do estator. Porém, a pequena altura do cilindro $(4 \mathrm{~cm})$ comprometeu o desempenho do motor quanto à sua rotação. No segundo teste, o rotor foi substituindo por outro de diâmetro um pouco menor de $12,5 \mathrm{~cm}$, mas com altura maior de $10 \mathrm{~cm}$, igualando a altura do estator. Com isso, foram conseguidos resultados bem mais satisfatórios. Com o novo rotor, o espaço entre o estator e rotor (entreferro) é de $1,5 \mathrm{~cm}$. É importante dizer que o entreferro deve ser o mínimo possível para obter o melhor desempenho do motor. A Fig. 14 mostra o rotor finalizado para o protótipo.

A Fig. 14a mostra a parte externa do rotor construindo com uma lata de leite em pó, e a Fig. 14b mostra o acoplamento feito com tarugo de nylon fixado na parte interna da lata, para o acoplamento do eixo. Numa segunda etapa do projeto, outro rotor foi construído considerando o modelo de gaiola de esquilo. A Fig. 15 mostra a nova
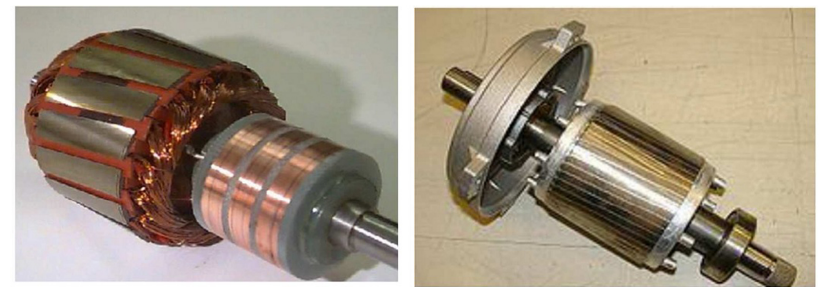

Figura 12: (a) O rotor bobinado e (b) o rotor em gaiola de esquilo. A bobina do rotor bobinado tem acesso por meio dos três anéis fixados no seu eixo.

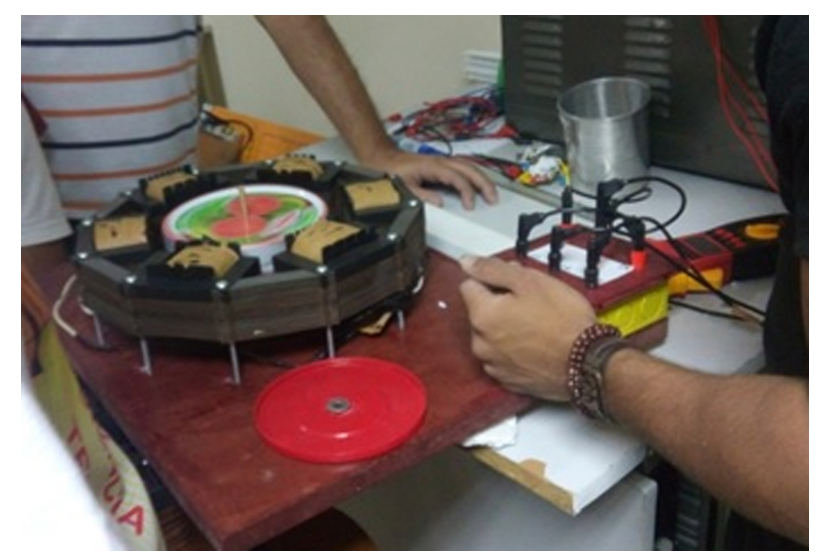

Figura 13: Testes da escolha do rotor.
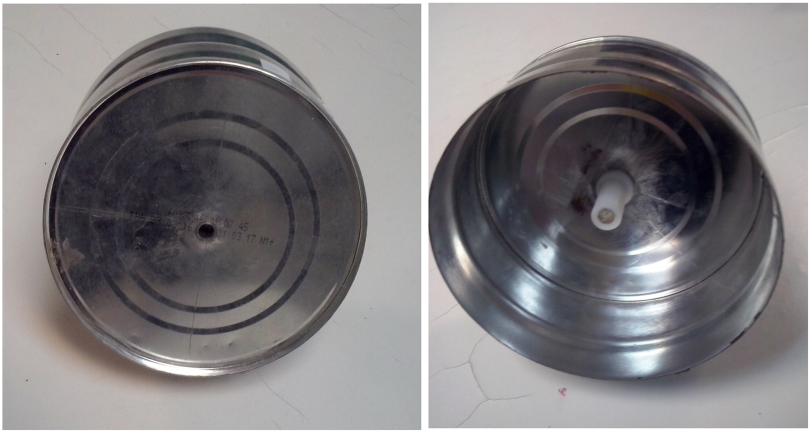

Figura 14: Proposta inicial do rotor do motor de indução.

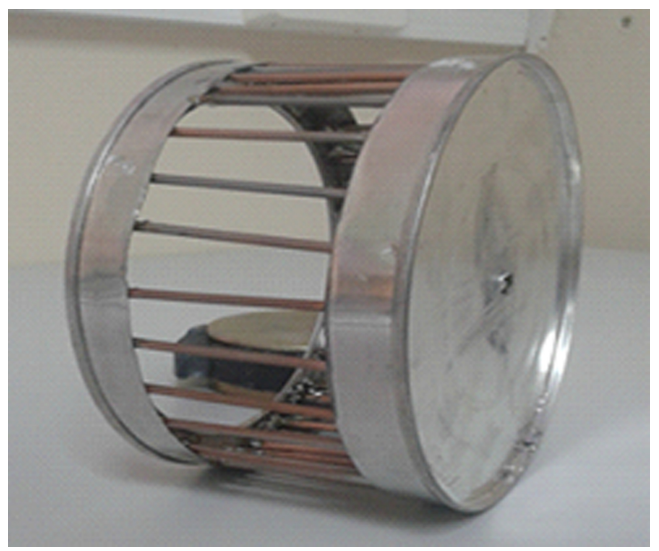

Figura 15: Rotor gaiola de esquilo finalizado.

versão do rotor com as barras condutoras e os dois anéis de curto-circuito.

A Fig. 15 mostra a semelhança do rotor com uma gaiola de esquilo, que dá origem ao seu nome. Os anéis de curto-circuito visto nas extremidades do rotor são feitos com as partes superior e inferior de uma lata para condicionamento de alimento. Esses anéis foram soldados com hastes condutoras aproveitadas de raios de roda de bicicleta. No rotor real mostrado na Fig. 12b, as barras são introduzidas em ranhuras no núcleo de ferro, formando uma peça única, rígida e compacta. No protótipo, não foi possível usar o núcleo de ferromagnético no rotor, devido a grande complexidade de construção das peças. Apesar desse problema, não houve nenhum prejuízo com o uso do protótipo para fins didáticos. A base do motor para o protótipo é construída de uma prancha de madeira de $(50 \times 50 \times 2) \mathrm{cm}$, e no centro da prancha é fixado um eixo metálico de diâmetro $0,5 \mathrm{~cm}$ para receber o rotor. Neste caso o eixo não gira junto com o rotor, diferentemente do rotor real que o eixo faz parte do rotor.

\section{Resultados}

O protótipo foi implementado e os testes foram satisfatórios. Algumas dificuldades foram surgidas ao decorrer do processo de construção do protótipo. A grande dificuldade foi a desmontagem do núcleo de ferromagnético do 
transformador, pois, devido o tempo longo de uso fez com as finas chapas ficassem bastante coladas, dificultando a retirada das mesmas. Outro problema foi no serviço de enrolar os fios nas bobinas. No início, o processo de bobinagem foi feito manualmente, sendo que as bobinas não apresentavam uniformidade, além disso, o tempo de finalização de uma bobinagem era muito grande e em várias vezes a contagem do número de voltas era perdida. Com a construção da bobinadeira e do contador de voltas, o processo teve expressivas melhorias de qualidade das bobinas e no tempo de trabalho. A Fig. 16 mostra o motor de indução aberto finalizado.

Quanto às competências no desenvolvimento da capacidade de investigação física, o protótipo possibilitou entender, por exemplo, o fenômeno eletromagnético do campo magnético girante do motor de indução. Para tanto, foi retido o rotor e colocado no centro do estator uma bússola ou mesmo um ímã posto numa superfície lisa. Ao energizar o motor a agulha imantada da bússola gira no sentido do campo, e ao inverter a posição de um dos terminais de ligação do motor, a agulha gira no sentido contrário. A Fig. 17 mostra o aparato do experimento.

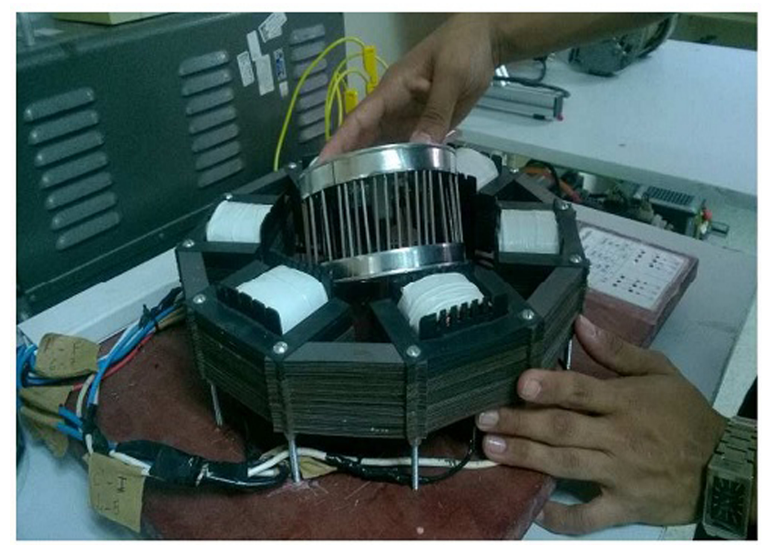

O protótipo do motor trifásico desenvolvido foi construído um caixa de terminais com seis bornes, possibilitando a ligação das bobinas nas configurações delta e estrela. Os testes foram realizados com tensões reduzidas usando um autotransformador. Os resultados são mostrados na placa de identificação do motor visto na Fig. 18.

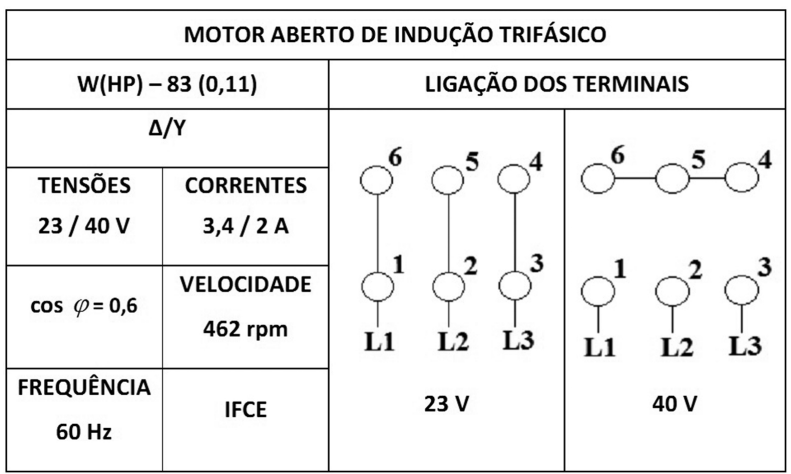

Figura 18: Placa de identificação do protótipo.

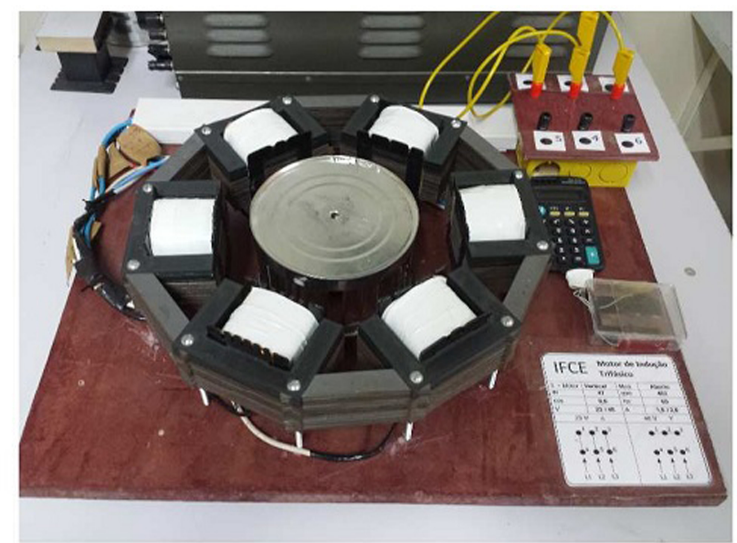

Figura 16: Motor de indução aberto gaiola de esquilo.

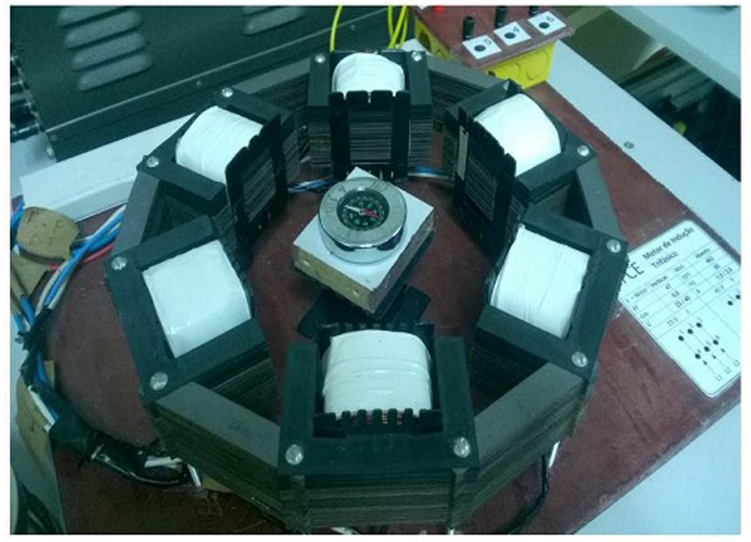

(a)

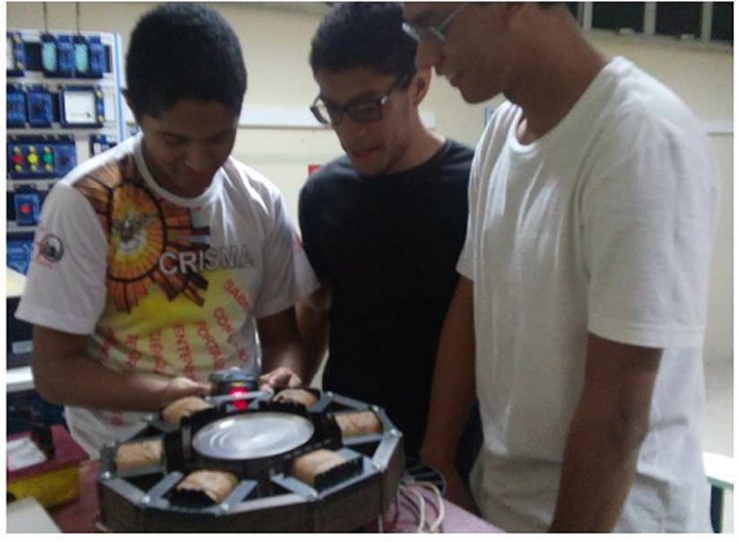

(b)

Figura 17: Testes do motor. (a) Demonstração do campo magnético girante, (b) e alunos fazendo a medição da velocidade do rotor com um tacômetro. 
Os testes foram realizados nas tensões de $23 \mathrm{~V}$ na ligação em delta $(\Delta)$ e de $40 \mathrm{~V}$ na ligação em estrela $(\mathrm{Y})$. Com essas tensões, as correntes foram de $3,4 \mathrm{~A}$ e $2 \mathrm{~A}$ para delta e estrela, respectivamente. Nessas condições de testes, a temperatura nas bobinas permaneceu numa faixa confortável de operação. A potência de entrada do motor foi de $83 \mathrm{~W}$ ou $0,11 \mathrm{CV}$. A potência de saída não foi possível determinar, devido à dificuldade de obter o torque mecânico no eixo do motor. $\mathrm{O}$ fator de potência encontrado foi de 0,6, e a velocidade de $462 \mathrm{rpm}$.

\section{Conclusões}

O protótipo do motor foi baseado nos primeiros motores de indução desenvolvido por Nikolas Tesla. Os diferenciais do protótipo são a facilidade de construção, a utilização de material de sucata, o baixo custo, e a facilidade de explicar fenômenos do eletromagnetismo que o motor experimenta. O baixo custo de construção possibilita que a maioria das escolas públicas possa reproduzir o protótipo e com isso equipar os seus laboratórios. Nas aulas práticas usando o motor, foram realizados vários experimentos, por exemplos, constatação do campo magnético nas bobinas, campo magnético girante, intensidade da força magnetomotriz, e corrente indutiva. Quanto aos aspectos didáticos-pedagógicos, o protótipo demonstrou-se um instrumento prático e fácil de ser aplicado nas aulas práticas. Nas aulas práticas das disciplinas de máquinas elétricas e de eletricidade e magnetismo dos cursos técnico em eletrotécnica e superior em automação industrial, percebeu-se na turma interesse e curiosidade dos alunos, observados, principalmente, a partir das inúmeras perguntas sobre o funcionamento do motor de indução aberto apresentado.

\section{Referências}

[1] V.F. Pinto, A.P. Viana e A.E.A. Oliveira, Revista Conexão Ponta Grossa 9, 84 (2013).

[2] N.A. Grandini e C.R. Grandini, Revista Brasileira de Ensino de Física 26, 251 (2004).

[3] A.T. Borges, Revista Brasileira de Ensino de Física 19, 291 (2002).

[4] M.R.L. Machado e P.R. Moreira, Educação profissional no Brasil, evasão escolar e transição para o mundo do trabalho, disponível em www.senept.cefetmg.br/ galerias/.../TerxaTema3Poster9.pdf, acessado em $22 / 06 / 2018$.

[5] K.M.C. Mattos e W.J.S. Perales, in Anais do Encontro Nacional de Engenharia de Produção (Rio de Janeiro, Brasil, 2008).

[6] W. Hauser, Introduction to the Principles of Electromagnetism (Addison-Wesley, Reading, 1971).

[7] R.P. Feynman, R.B. Leighton e M. Sands, Lições de Física de Feynman (Bookman, Porto Alegre, 2008), v. 2.

[8] D. Halliday, R. Resnick e J. Walker, Fundamentos de física III (LTC, Rio de Janeiro, 2009), v. 4.

[9] M.N.O. Sadiku, Elements of Electromagnetism (Oxford University Press, Nova Iorque, 2007), p. 765.
[10] S.J. Chapman. Fundamentos de máquinas elétricas (AMGH, Porto Alegre, 2014), p. 700.

[11] S.D. Umans, Máquinas elétricas de Fitzgerald e Kingsley (AMGH, Porto Alegre, 2014), p. 708.

[12] I. Barbi, SBA: Controle \& Automação 2, 1 (1988).

[13] G.C. Nascimento Junior, Máquinas elétricas: teoria e ensaios (Érica, São Paulo, 2011), p. 259.

[14] A. Martignoni, Transformadores (Globo, São Paulo, 1991). 